\title{
Erythrocyte sialic acid content during aging in humans: Correlation with markers of oxidative stress
}

\author{
Mohammad Murtaza Mehdi, Prabhakar Singh and Syed Ibrahim Rizvi* \\ Department of Biochemistry, University of Allahabad, Allahabad, India
}

\begin{abstract}
Sialic acids are substituted neuraminic acid derivatives which are typically found at the outermost end of glycan chains on the membrane in all cell types. The role of erythrocyte membrane sialic acids during aging has been established however the relationship between sialic acid and oxidative stress is not fully understood. The present work was undertaken to analyze the relationship between erythrocyte membrane sialic acid with its plasma level, membrane and plasma lipid hydroperoxide levels and plasma total antioxidant capacity. Results show that sialic acid content decreases significantly $(P<0.001)$ in RBC membrane $(r=-0.901)$ and increases in plasma $(r=0.860)$ as a function of age in humans. Lipid peroxidation measured in the form of hydroperoxides increases significantly $(P<0.001)$ in plasma $(r=0.830)$ and RBC membranes $(r=0.875)$ with age in humans. The Trolox Equivalent Total Antioxidant Capacity (TETAC) of plasma was found to be significantly decreased $(P<0.001, r=-0.844)$. We observe significant correlations between decrease of erythrocyte membrane sialic acid and plasma lipid hydroperoxide and TETAC. Based on the observed correlations, we hypothesize that increase in oxidative stress during aging may influence the sialic acid decomposition from membrane thereby altering the membrane configuration affecting many enzymatic and transporter activities. Considering the importance of plasma sialic acid as a diagnostic parameter, it is important to establish age-dependent reference.
\end{abstract}

Keywords: Erythrocyte, aging, sialic acid, oxidative stress

\section{Introduction}

The plasma membrane performs the important cellular function of maintaining cellular integrity and restricting traffic between inside and outside the cell. The variety of intracellular metabolic requirements and extracellular signals necessitate the membrane to be extremely responsive and dynamic [12]. Such functions are only possible when the membrane's chemical composition is stable and the complex infrastructure remains intact throughout the organism's lifespan [6]. The deterioration of membrane integrity is thought to be a major cause of the aging process [11]. The human

*Corresponding author: Dr. Syed Ibrahim Rizvi, Department of Biochemistry, University of Allahabad, Allahabad, 211002, India. Tel.: +91 9415305910; E-mail: sirizvi@gmail.com. erythrocyte with its life span of 120 days provides a good model system to study age-dependent alterations in humans [17]. The erythrocyte is continuously exposed to oxygen making it susceptible to oxidation mediated damage [34,35]. Several age-dependent changes in erythrocyte biological membrane constituents in humans have been reported [17]. Alterations in the activity of plasma membrane redox system [44], AFR reductase [41], acetylcholinesterase [34], protein [18,44] and lipid oxidation [31] have already been reported.

As essential constituents of many glycoproteins, glycopeptides and glycolipids, the naturally occurring sialic acids are substituted neuraminic acid derivatives among which the most important is $\mathrm{N}$ acetylneuraminic acid, NANA (5 amino-3,5-dideoxyD-nonulosonic acid) typically found at the outermost end of glycan chains of all cell types $[1,26]$. These nine-carbon backbone acidic sugars adorn all cell sur- 
faces and most secreted proteins of vertebrates and few higher invertebrates, mediating or modulating a variety of normal and pathological processes [2]. Due to negative charge and hydrophobicity, sialic acids have many structural and modulatory roles. They affect the action of some hormones, the recognition of different compounds, the cellular adhesiveness, the catalytic properties of enzymes, the transport process and antigenicity [36]. They serve as components of binding sites for various pathogens and toxins $[8,10]$. Sialic acid specific lectins found in invertebrates are supposed to be involved in defense against microbes expressing sialic acid on their surface [37]. Thus changes in sialic acid content may lead to possible topological structural change in the membrane which may also affect the body's defense mechanism against microbes. The loss of sialic acid during red cell senescence has already been reported and has been hypothesized to contribute to the clearance of erythrocyte from the circulation at the end of its life span [4,7].

Based on Harman's free radical theory of aging, several reports have provided enough evidence that the erythrocyte oxidative stress parameters significantly correlate with the plasma membrane redox potential $[3$, 43]. In view of several recent findings reporting alterations in erythrocyte membrane structure and composition as a function of human age [27,47], this study was undertaken to determine sialic acid levels in erythrocyte membrane and plasma during human aging. Membrane lipid peroxidation is also a major factor in the pathogenesis of many disease states including aging $[31,39,42]$ and some reports show a link between blood malondialdehyde levels and sialic acid [19,33], we also correlate the sialic acid levels with lipid peroxidation index and also with plasma total antioxidant capacity.

\section{Materials and methods}

The study was carried out on 81 normal healthy subjects of both sexes (54 males and 27 females) belonging to urban middle class background between the ages of 21 and 90 years. Young volunteers were mainly from University while older subjects were relatives or acquaintances of faculty or students. The criteria for selection of subjects were the same as reported earlier [18], the basic biomedical parameters recorded were (range) BMI: $18.5-26.4 \mathrm{~kg} / \mathrm{m} 2$, blood glucose fasting: 64.8-90.0 mg/dL, postprandial $104.4-140.0 \mathrm{mg} / \mathrm{dL}$, blood pressure systolic: $90-142 \mathrm{~mm} \mathrm{Hg}$, diastolic: $60-$
$92 \mathrm{~mm} \mathrm{Hg}$. All volunteers were screened for asthma, tuberculosis, diabetes mellitus or any other major illness. None of the subjects were smokers or were taking any medication. Care was also taken to exclude volunteers taking/have taken the any nutritional supplements since last three months. The elderly subjects were living at home but functionally independent without any cognitive impairment. All persons gave their informed consent for the use of their blood samples for the study. Venous blood was obtained at fasting condition in the morning by venipuncture in sterile polystyrene tubes containing heparin. Plasma was obtained by centrifuging the blood at $800 \mathrm{xg}$ for $10 \mathrm{~min}$ at $4^{\circ} \mathrm{C}$. Antioxidant capacity measurements were performed immediately, for further analyses the plasma was frozen and stored at $-80^{\circ} \mathrm{C}$ for not more than $2-3$ days. The protocol of study was in conformity with the guidelines of the Allahabad University Ethical Committee.

\subsection{Isolation of erythrocyte membranes}

Preparation of erythrocyte membranes (ghosts) was carried out according to the method of Dodge et al. [16] with slight modifications. Briefly, washed and packed erythrocytes were lysed by adding 10 volume of $5 \mathrm{mM}$ phosphate buffer $\mathrm{pH} 7.4$ (at $4^{\circ} \mathrm{C}$ ) while mixing. After leaving on ice for $30 \mathrm{~min}$, the erythrocyte membranes were packed by centrifugation at 20,000 $\mathrm{g}$ for $10 \mathrm{~min}$ at $4^{\circ} \mathrm{C}$ and the hemoglobin-containing supernatant was removed. The erythrocyte membranes were then washed three times by suspending in fresh buffer (same volume as used for lysis) followed by centrifugation under the same conditions. Finally, the membranes were suspended in hypotonic $5 \mathrm{mM}$ buffer (same volume as used for lysis) followed by centrifugation under the same conditions and then resuspended in hypotonic $5 \mathrm{mM}$ phosphate buffer $\mathrm{pH}$ 7.4. Erythrocyte membranes were quantified on the basis of protein concentration using the assay of Lowry et al. [29].

\subsection{Determination of membrane and plasma sialic acid levels}

It was performed by the method proposed by Spyridaki et al. [26]. To determine the sialic acid level in membrane/plasma, $0.10 \mathrm{ml}$ of $0.04 \mathrm{M}$ periodic acid was added to a glass tube containing $500 \mu$ l diluted (20 times) sample solution. It was mixed thoroughly and allowed to stand in ice bath for $30 \mathrm{~min}$. Thereafter $1.25 \mathrm{ml}$ of resorcinol working solution $(5 \mathrm{ml}$ of $6.0 \%$ resorcinol solution, $0.125 \mathrm{ml}$ of $0.1 \mathrm{M}$ copper sulphate 
solution and $19.875 \mathrm{ml}$ of distilled water, brought to a final volume of $50 \mathrm{ml}$ with $10 \mathrm{M} \mathrm{HCl}$ ) was added, mixed and heated at $98^{\circ} \mathrm{C}$ for $5 \mathrm{~min}$. Tubes were cooled in an ice bath for approximately $2 \mathrm{~min}$. Lastly $3.25 \mathrm{ml}$ of $n$-butanol was added. The solutions were mixed vigorously and the tubes were placed in a water bath at $37^{\circ} \mathrm{C}$ for at $3 \mathrm{~min}$ for colour to stabilize. Immediately after removing the solutions from the water bath their absorbance were measured at $625 \mathrm{~nm}$ against a reagent blank. A calibration graph was prepared with standard solutions of NANA in the range 20-200 $\mu \mathrm{M}$ and the unknown concentrations of total sialic acid in samples were calculated. Sialic acid level in membrane is reported in terms of $\mu \mathrm{g} / \mathrm{mg}$ membrane protein. Plasma sialic acid is measured as $\mu \mathrm{M}$.

\subsection{Determination of lipid hydroperoxides in $R B C$ membranes and plasma}

It was done by the method proposed by Firuzi et al. [28]. For measuring ROOHs $200 \mu \mathrm{L}$ of diluted (4 times) plasma/200 $\mu \mathrm{l}$ membrane ( 0.8 to $1.0 \mathrm{mg}$ protein $/ \mathrm{ml}$ ) was taken in duplicate tubes. In one of the tubes was added $20 \mu \mathrm{L}$ triphenylphosphine (methanolic) and to another $20 \mu \mathrm{L}$ of methanol. The tubes were vortex-mixed every $10 \mathrm{~min}$ and incubated at room temperature in the dark for $30 \mathrm{~min}$. After completion of incubation period $1800 \mu \mathrm{l}$ of FOX solution (Solution A: $4.4 \mathrm{mM}$ butylated hydroxytoluene in methanol, Solution B: $1 \mathrm{mM}$ xylenol orange and $2.5 \mathrm{mM}$ ammonium ferrous sulfate dissolved in sulfuric acid $250 \mathrm{mM}$. Working solution was prepared by mixing A and B solutions at a proportion of 9: 1 respectively) was added in both tubes. The samples were again incubated for $1 \mathrm{hr}$ at room temperature in the dark with vortex-mixing at every $10 \mathrm{~min}$. After second incubation samples were centrifuged at 16,000 $\mathrm{g}$ for 7 minutes and absorbance of the supernatants was measured at $560 \mathrm{~nm}$. The absorbance of the samples treated with TPP was subtracted from non-treated samples to calculate the concentration of ROOHs. Results were calculated with the aid of a calibration curve using hydrogen peroxide in the range $1-10 \mu \mathrm{M}$. Plasma $\mathrm{RCOOH}$ is expressed as $\mathrm{mM}$ in and membrane $\mathrm{RCOOH}$ is expressed as $\mathrm{nmol} / \mathrm{mg}$ membrane protein.

\subsection{Determination of total antioxidant capacity by CUPRAC method}

It was done by method proposed by Apak et al. [32]. $500 \mu \mathrm{l}$ Copper (II) Chloride solution $\left(1.0 \times 10^{-2} \mathrm{M}\right)$,
$500 \mu \mathrm{l}$ Neocuproine solution $\left(7.5 \times 10^{-3} \mathrm{M}\right)$ and $500 \mu \mathrm{l}$ Ammonium Acetate buffer (1.0 M, pH 7.0) was added to $2.50 \mathrm{ml}$ diluted plasma (dilution 1:25) and final absorbance was measured at $450 \mathrm{~nm}$. Total Antioxidant Capacity was measured by comparing absorbance with Trolox standard curve between 20-200 $\mu \mathrm{M}$.

\subsection{Statistical analysis}

Statistical analysis was done by using the software GraphPad Prism 5 version 5.01. To assess relationships between parameters, Pearson's correlation coefficient (r) was derived at $95 \%$ confidence interval by taking the $P$ value $<0.05$ as significant.

\section{Results}

The age dependent study was conducted on blood samples obtained from normal healthy persons of both sexes between the ages 21 to 90 years. Our observations show that erythrocyte membrane sialic acid level decreases as a function of age (Fig. 1, Pearson's $\left.r=-0.901, P<0.001, \mathrm{r}^{2}=0.788\right)$. The plasma shows increasing levels of sialic acids constituents with age (Fig. 2, Pearson's $r=0.860, P<0.001, r^{2}=$ $0.740)$.

Levels of lipids hydroperoxides in erythrocyte membrane and plasma increased significantly with age (Fig. 3, Pearson's $r=0.875, P<0.001, r^{2}=0.765$ and Fig. 4, Pearson's $r=0.830, P<0.001, r^{2}=$ 0.688).

The trolox equivalent antioxidant capacity (TEAC) is defined as the millimolar concentration of a trolox solution having the antioxidant capacity equivalent to a $1.0 \mathrm{mM}$ solution of the substance under investigation. The total antioxidant capacity (TAC) of plasma measured by CUPRAC methods (mentioned as Trolox Equivalents Total Antioxidant Capacity, TETAC-CUPRAC) shows significant negative correlation with age (Fig. 5, Pearson's $r=-0.844, P<$ $\left.0.001, r^{2}=0.609\right)$.

Based on our results, we also show correlations between sialic acid content and markers of oxidative stress as a function of human age: erythrocyte membrane sialic acid and lipid hydroperoxide (Fig. 6a) and plasma sialic acid and total plasma antioxidant capacity (Fig. 6b).

\section{Discussion}

The erythrocyte membrane consists of a lipid bilayer composed of $50 \%$ protein, $40 \%$ lipid, and $10 \%$ car- 


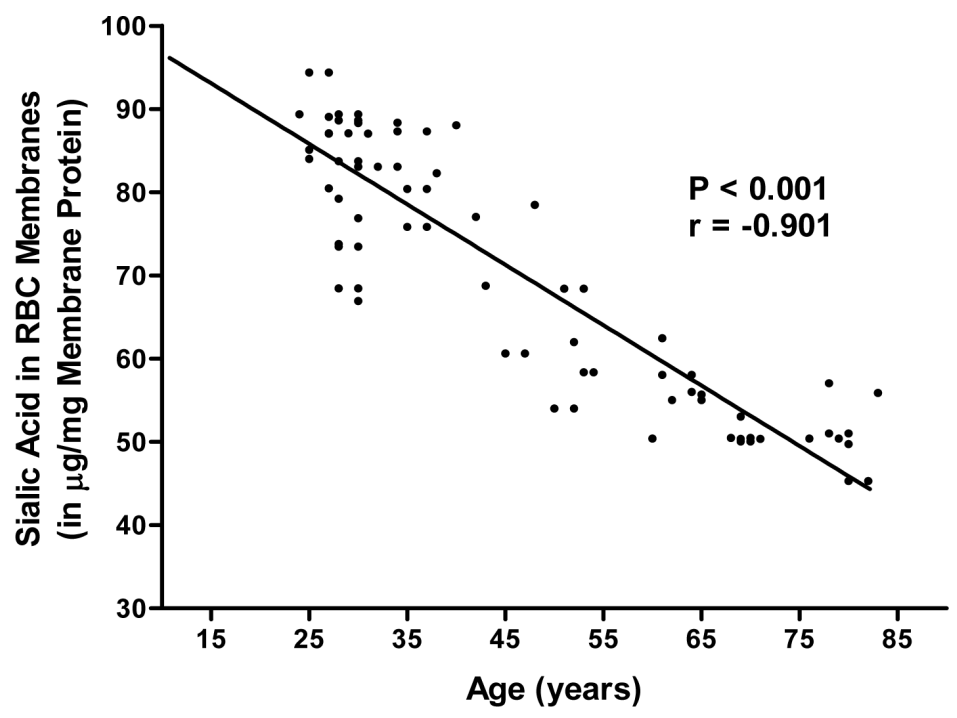

Fig. 1. Sialic acid content in RBC membranes (in $\mu \mathrm{g} / \mathrm{mg}$ membrane protein) as a function of human age.

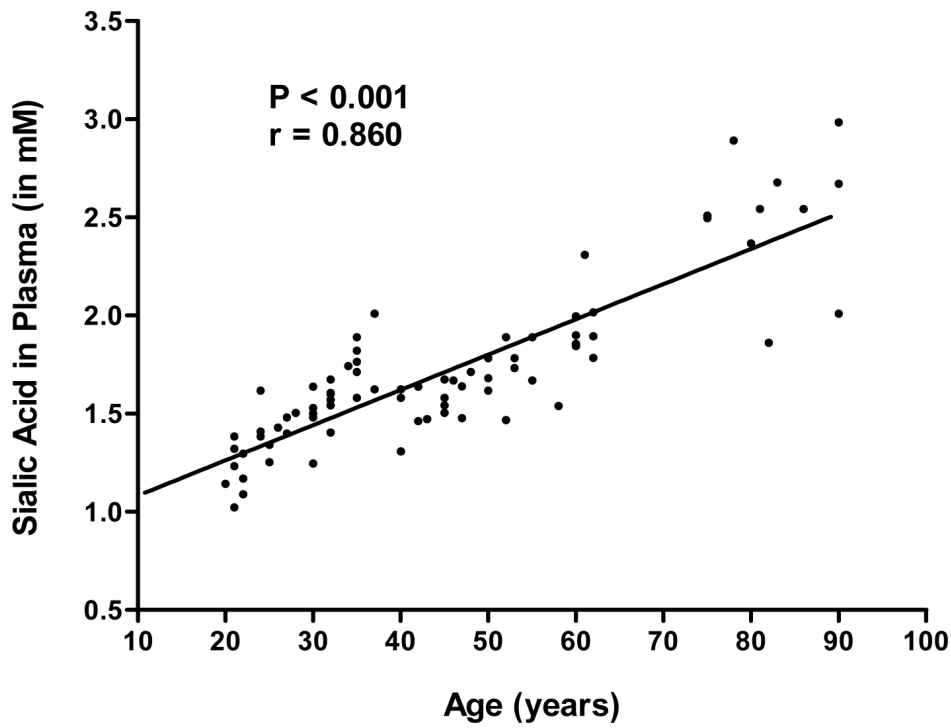

Fig. 2. Plasma sialic acid (in $\mathrm{mM}$ ) as a function of human age.

bohydrate. The human erythrocyte membrane is continuously exposed to oxidative stress in the circulation and undergoes various modifications of cellular components. The study of plasma membrane alterations during human aging might provide insight on the biological mechanisms associated with longevity and aging. Our results show that sialic acid, the carbohydrate moiety of glycoproteins, is detached or degraded from erythrocyte membranes at a higher rate in older individuals compared to younger individuals, the process shows significant $(P<0.001)$ negative correlation $(r=-0.901)$ with membrane lipid hydroperox- idation. Importantly, sialic acid contributes negative charge on human erythrocytes and other cell types providing charge repulsion and prevention of unwanted interactions of cells in the blood circulation. Thus loss of sialic acid from the membrane may result in change in surface charge thereby causing altered biophysical properties of the red cell membrane.

Sialic acid molecules have persisted on all cell types in all vertebrates for a long evolutionary time and over the last few decades several sialic acid binding proteins have been discovered $[1,8,10]$. One of the most important functions is 'molecular mimicry', in which suc- 


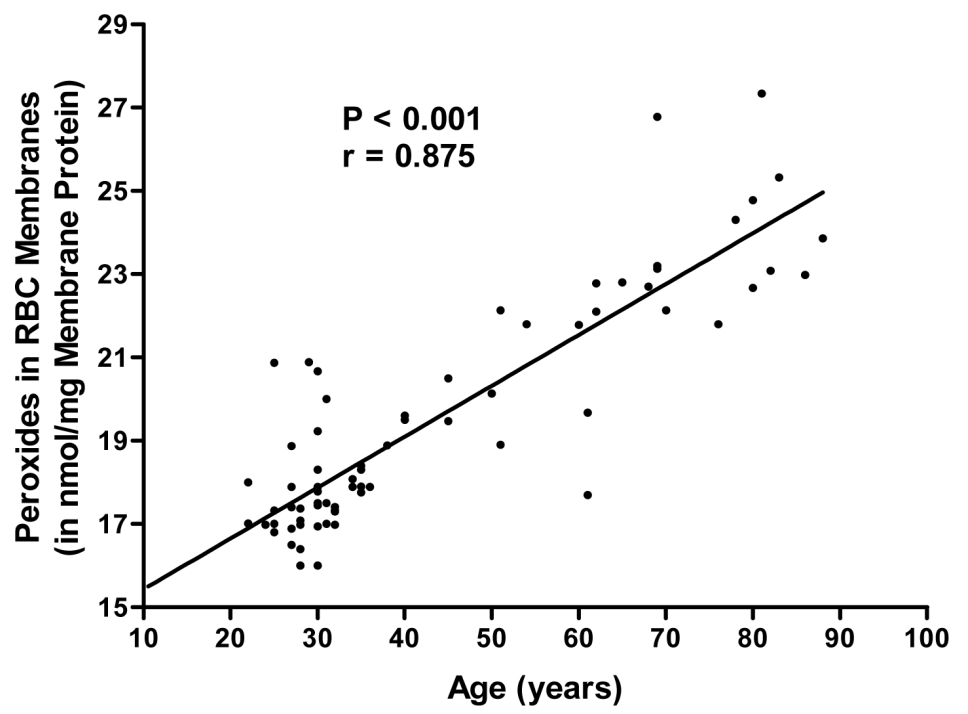

Fig. 3. Lipid hydroperoxides in RBC membranes (in nmol/mg membrane protein) as a function of human age.

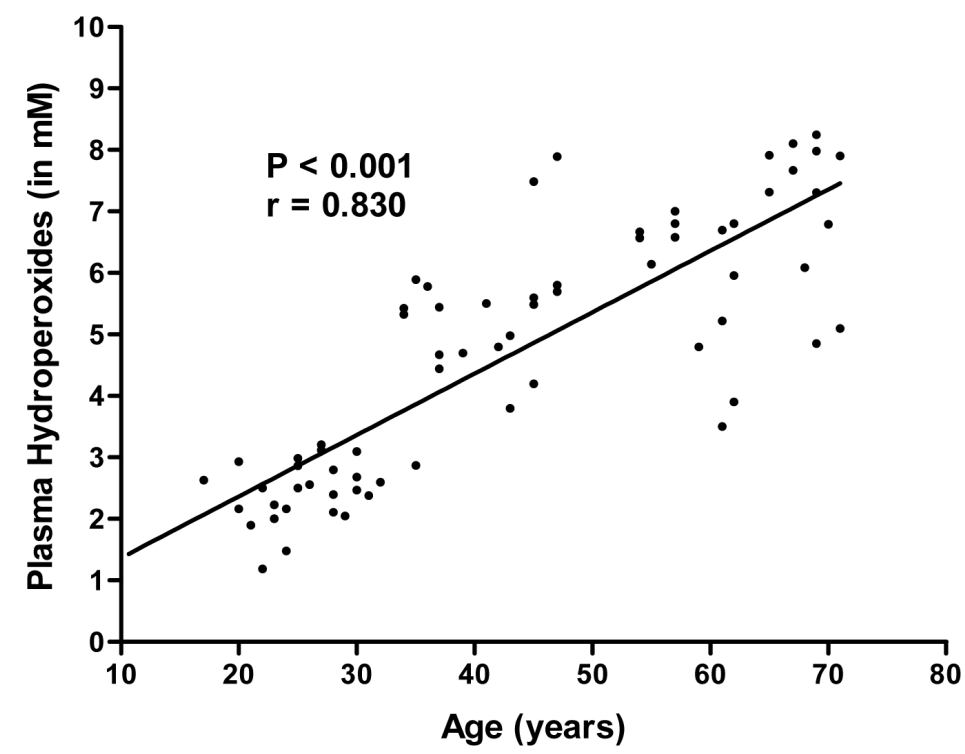

Fig. 4. Plasma lipid hydroperoxide levels (in $\mathrm{mM}$ ) as a function of human age.

cessful microbial pathogens decorate themselves with sialic acids, assisting in evasion of host immunity [9]. Sialic acid containing high molecular weight glycoprotein mucin, which functions as hydroxyl radical scavenger, loses its ability when desialylated, thus mucin has the ability to protect cells from oxidants [46]. Since oxidative stress is known to increase with age $[39,40]$, It may also be presumed that loss of sialic acid could contribute to decreased ability of mucin to protect against oxidants.

We find that plasma sialic acid levels increase as a function of age in humans. Significantly sialic acid level in plasma is not only due to loss from the RBC membrane through oxidative damage but also due to damages in the various organ cells. Sialic acid measurements in blood have been used to predict disease risk in humans [15,24,25]. The plasma sialic-acid index of Apo $\mathrm{J}$ (moles of sialic acid per mole of Apo $\mathrm{J}$ protein) is used as a marker for early detection of chronic alcohol consumption [30]. The plasma sialic acid level has also been considered as an important parameter in identifying subjects prone to develop type-2 diabetes [21]. 


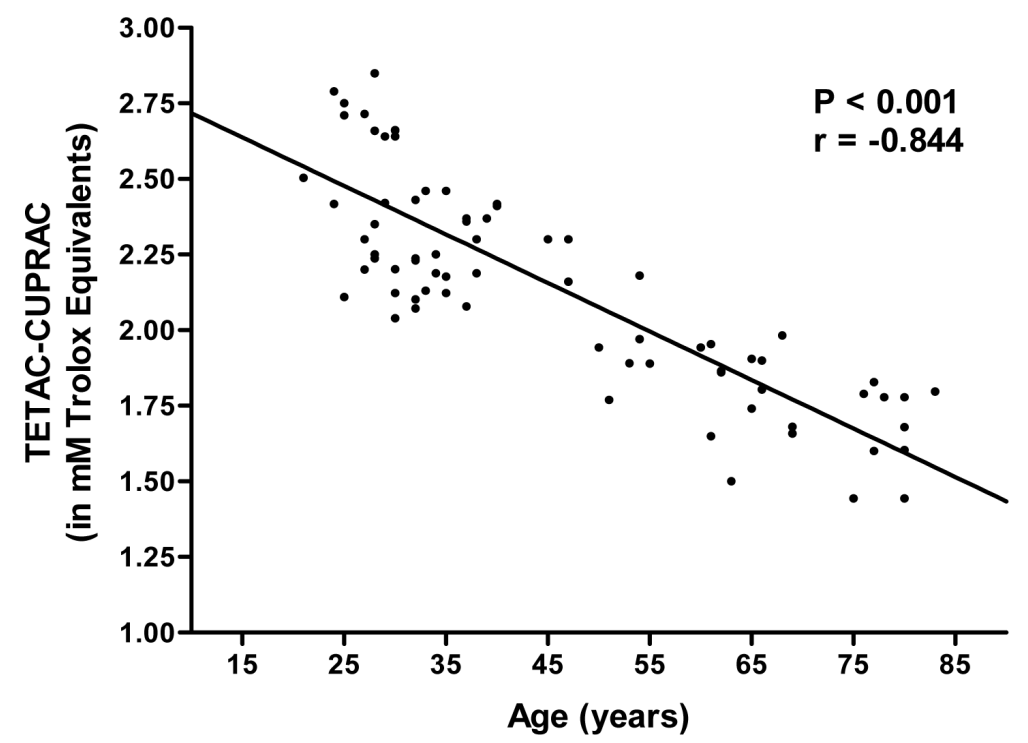

Fig. 5. Trolox equivalent total antioxidant capacity (in $\mathrm{mM}$ trolox equivalents) of plasma plotted as a function of human age.

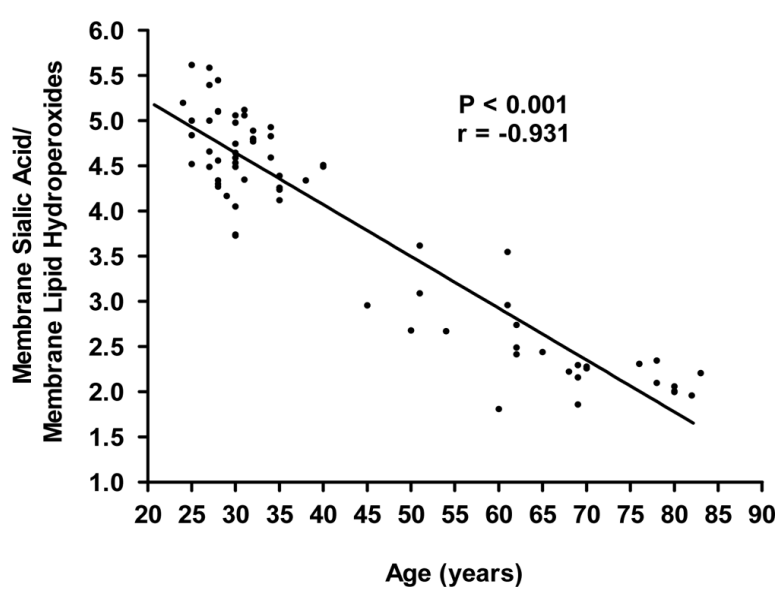

(a)

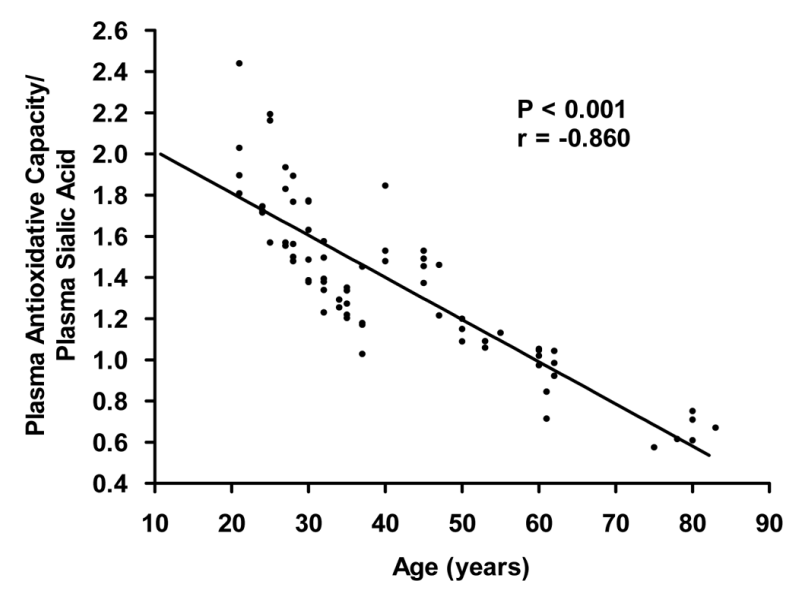

(b)

Fig. 6. a) Plot of quotient (membrane sialic acid level/ membrane lipid hydroperoxides) as a function of human age. b) Plot of quotient (plasma sialic acid level/ antioxidant capacity of plasma) as a function of human age.

Patients with hypertension have decreased membrane sialic acid compared to normotensive subjects and this shows that decrease in sialic acid content may also influence the rheological properties of blood [4]. Significantly low sialic acid erythrocyte concentrations with contemporary high sialic acid plasma levels have been considered as markers of ischemic stroke [20]. Levels of serum total sialic acid in cholangiocarcinoma patients are found higher than the levels in healthy individuals [38]. Thus a higher plasma sialic acid level in older persons may be contributing to the development of several age-dependent diseases.
The FOX assay originally proposed by NouroozZadeh et al. [13] is a simple spectrophotometric method that represents an alternative to more sophisticated and time consuming methodologies for measurement of hydroperoxides (ROOHs) in biological fluids. Mean plasma hydroperoxide $(\mathrm{ROOH})$ concentrations in our subjects of different age groups corroborate previous observations [13] and our results showed that there was a significant $(P<0.001)$ increase in the mean concentration of ROOHs in erythrocyte membranes as well as in plasma with increasing age. The correlation of membrane sialic acid level with membrane lipid hydroper- 
oxidation (Fig. 6a) provide further basis to the argument that altered redox potential during aging causes loss of sialic acid from the erythrocyte membrane. The importance of our study on human blood parameters is highlighted in the context of report that blood levels of lipid peroxides provide a reliable indication about the redox status in other tissues including skeletal muscle, heart and liver [5].

The increase in oxidative stress and inflammatory mediators during aging has been linked to the levels of advanced glycation end products (AGE's) [23, 45]. These prooxidant factors (AGE's) specially $\mathrm{N} \varepsilon$ carboxymethyl-lysine may also contribute to our observed changes in sialic acid level and lipid peroxidation levels during aging [14]. A decrease in total plasma total antioxidant capacity as a function of human age has been reported by us and by other workers [22, 39] however in a significant finding we showed that the decrease of antioxidant potential was sharper in case of Indian population. Our present observations corroborate earlier reports. The significant negative correlation (Fig. 6b, $r=-0.860$ ) between plasma TAC and plasma sialic acid reflects the relationship between redox state of plasma and sialic acid, however, the mechanism of relationship is still not clear.

In conclusion, the increase in oxidative stress during aging causes increased sialic acid decomposition from erythrocyte membrane which may lead to altered biophysical property of the membrane affecting many enzymatic and transporter activities. Considering the importance of plasma sialic acid as a diagnostic parameter, it is important to establish age-dependent reference. The questions about the native conformation of sialic acid after detachment, the amount, rate and the mechanism of sialic acid removal; and how the increased plasma sialic acid levels are maintained during aging however remain unanswered.

\section{Acknowledgements}

Mohammad Murtaza Mehdi is a recipient of Senior Research Fellowship from Indian Council of Medical Research, New Delhi, India. Financial assistance from University Grants Commission in the form of MRP F 39-392/2009 to SIR is gratefully acknowledged.

\section{References}

[1] A. Varki, Glycan-based interactions involving vertebrate sialic-acid-recognizing proteins, Nature 446 (2007), 10231029.
[2] A. Varki, Sialic acids in human health and disease, Trends in Molecular Medicine 14 (2008), 351-360.

[3] A.D.N.J. de Grey, The plasma membrane redox system: a candidate source of aging-related oxidative stress, Age 27 (2005), 129-138.

[4] A.L. Hadengue, M. Del-Pino, A. Simon and J. Levenson, Erythrocyte disaggregation shear stress, sialic acid, and cell aging in humans, Hypertension 32 (1998), 324-330.

[5] A.S. Veskoukis, M.G. Nikoliadis, A. Kyparos and D. Kouretas, Blood reflects tissue oxidative stress depending on biomarker and tissue studied, Free Radical Biology and Medicine 47 (2009), 1371-1374.

[6] B.P. Yu, Oxidative damage by free radicals and lipid peroxidation in aging, in: Free Radicals in Aging, B.P. Yu, ed., CRC Press, Boca Raton, 1993, pp. 57-88.

[7] D. Bratosin, J. Mazurier, H. Debray, M. Lecocq, B. Boilly, C. Alonso, M. Moisei, C. Motas and J. Montreuil, Flow cytofluorimetric analysis of young and senescent human erythrocytes probed with lectins. Evidence that sialic acids control their life span, Glycoconjugate Journal 12 (1995), 258-267.

[8] D. Ilver, P. Johansson, H. Miller-Podraza, P.G. Nyholm, S. Teneberg and K.A. Karlsson, Bacterium-host proteincarbohydrate interactions, Methods in Enzymology 363 (2003), 134-157.

[9] E.R. Vimr, K.A. Kalivoda, E.L. Deszo and S.M. Steenbergen, Diversity of microbial sialic acid metabolism, Microbiology and Molecular Biology Reviews 68 (2004), 132-153.

[10] F. Lehmann, E. Tiralongo and J. Tiralongo, Sialic acid-specific lectins: occurrence, specificity and function, Cellular and Molecular Life Sciences 63 (2006), 1331-1354.

[11] G. Spiteller, Are changes of the cell membrane structure causally involved in the aging process? Annals of New York Academy of Sciences 959 (2002), 30-44.

[12] G. Vereb, J. Szollosi, J. Matko, P. Nagy, T. Farkas, L. Vigh, L. Matyus, T.A. Waldmann and S. Damjanovich, Dynamic, yet structured: The cell membrane three decades after the Singer-Nicolson model, Proceedings of National Academy of Science, USA 100 (2003), 8053-8058.

[13] J. Nourooz-Zadeh, J. Tajaddini-Sarmadi, S. McCarthy, D.J. Betteridge and S.P. Wolff, Elevated levels of authentic plasma hydroperoxides in NIDDM, Diabetes 44 (1995), 1054-1058.

[14] J. Uribarri, WJ Cai, M. Peppa, S. Goodman, L Ferrrucci, G. Striker and H. Vlassara, Circulating glycotoxins and dietary advanced glycation endproducts: two links to inflammatory response, oxidative stress, and aging, Journal of Gerontology Series A: Biological Science and Medical Science 2 (2007), 427-433.

[15] J.C. Pickup, M.B. Mattock, M. A. Crook, G.D. Chusney, D. Burt and A.P. Fitzgerald, Serum sialic acid concentration and coronary heart disease in NIDDM, Diabetes Care 18 (1995), 1100-1103.

[16] J.T. Dodge, C. Mitchell and D.J. Hanahan, The preparation and chemical characteristics of hemoglobin-free ghosts of human erythrocytes, Archives of Biochemistry and Biophysics 100 (1963), 119-130.

[17] K.B. Pandey and S.I. Rizvi, Markers of oxidative stress in erythrocytes and plasma during aging in humans, Oxidative Medicine and Cellular Longevity 3 (2010), 2-12.

[18] K.B. Pandey, M.M. Mehdi, P.K. Maurya and S.I. Rizvi, Plasma protein oxidation and its correlation with antioxidant potential during human aging, Disease Markers 29 (2010), 31-36.

[19] K.M. Surapaneni and V. Vishnu Priya, Altered serum total sialic acid, lipid peroxidation, ceruloplasmin and glutathione 
reductase levels in patients with carcinoma of prostate, Journal of Clinical and Diagnostic Research 3 (2009), 1483-1485.

[20] L. Nanetti, A. Vignini, F. Raffaelli, R. Taffi, M. Silvestrini, L. Provinciali and L. Mazzanti, Sialic acid and sialidase activity in acute stroke, Disease Markers 25 (2008), 167-173.

[21] M. Gavella, V. Lipovac, A. Car, M. Vucic, L. Sokolic and R. Rakos, Serum sialic acid in subjects with impaired glucose tolerance and in newly diagnosed type 2 diabetic patients, Acta Diabetologica 40 (2003), 95-100.

[22] M.L. Alexandrova and P.G. Bochev, Reduced extracellular phagocyte oxidative activity, antioxidant level changes and increased oxidative damage in healthy human blood as a function of age, Age 31 (2009), 99-107.

[23] M. Peppa, J. Uribarri and H. Vlassara, Aging and glycoxidant stress, Hormones 7 (2008), 123-133.

[24] M. Ponnio, H. Alho, S.T. Nikkari, U. Olsson, U. Rydberg and P. Sillanaukee, Serum sialic acid in a random sample of the general population, Clinical Chemistry 45 (1999), 1842-1849.

[25] M.A. Crook, K. Earle, A. Morocutti, J. Yip, G. Viberti and J.C. Pickup, Serum sialic acid, a risk factor for cardiovascular disease, is increased in IDDM patients with microalbuminuria and clinical proteinuria, Diabetes Care 17 (1994), 305-310.

[26] M.H.E. Spyridaki and P.A. Siskos, An improved spectrophotometric method for the determination of free, bound and total $\mathrm{N}$-acetylneuraminic acid in biological fluids, Analytica Chimica Acta 327 (1996), 277-285.

[27] M.V. Kameneva, K.O. Garrett, M.J. Watach and H.S. Borovetz, Red blood cell aging and risk of cardiovascular diseases, Clinical Hemorheology and Microcirculation 18 (1998), 67-74.

[28] O. Firuzi, P. Mladenka, V. Riccieri, A. Spadaro, R. Petrucci, G. Marrosu and L. Saso, Parameters of oxidative stress status in healthy subjects: their correlations and stability after sample collection, Journal of Clinical Laboratory Analysis 20 (2006), $139-148$.

[29] O.H. Lowry, N.J. Rosebrough, A.L. Farr and R.J. Randall, Protein measurement with the Folin Phenol reagent, Journal of Biological Chemistry 193 (1951), 265-275.

[30] P. Ghosh, E.A. Hale and M.R. Lakshman, Plasma sialic-acid index of apolipoprotein $\mathrm{J}$ (SIJ): a new alcohol intake marker, Alcohol 25 (2001), 173-179.

[31] P.K. Maurya and S.I. Rizvi, Protective role of tea catechins on erythrocytes subjected to oxidative stress during human aging, Natural Product Research 23 (2009), 1072-1079.

[32] R. Apak, K. Guclu, M. Ozyurek, S.E. Karademir and M. Altun, Total antioxidant capacity assay of human serum using copper(II)-neocuproine as chromogenic oxidant: The CUPRAC method, Free Radical Research 39 (2005), 949961.

[33] R. Iijima, T. Ichikawa and M. Yamazaki, Sialic acid attenuates the cytotoxicity of the lipid hydroperoxides HpODE and HpETE, Carbohydrate Research 344 (2009), 933-935.
[34] R. Jha and S.I. Rizvi, Age-dependent decline in erythrocyte acetylcholinesterase activity: correlation with oxidative stress, Biomedical Papers 153 (2009), 195-198.

[35] R. Jha and S.I. Rizvi, Carbonyl formation in erythrocyte membrane proteins during aging in humans, Biomedical Papers 155 (2011), 39-42.

[36] R. Schauer, Chemistry, metabolism and biological functions of sialic acid, Advances in Carbohydrate Chemistry and Biochemistry 40 (1982), 131-234.

[37] S. Tunkijjanukij, H. Giaever, C.C. Chin and J.A. Olafsen, Sialic acid in hemolymph and affinity purified lectins from two marine bivalves, Comparative Biochemistry and Physiology Part B, Biochemistry and Molecular Biology 119 (1998), 705713.

[38] S. Wongkhama, C. Boonlaa, S. Kongkhamb, C. Wongkhama, V. Bhudhisawasdic and B. Sripad, Serum total sialic acid in cholangiocarcinoma patients: an ROC curve analysis, Clinical Biochemistry 34 (2001), 537-541.

[39] S.I. Rizvi and P.K. Maurya, Markers of oxidative stress in erythrocytes during aging in humans, Annals of New York Academy of Sciences 1100 (2007), 373-382.

[40] S.I. Rizvi and P.K. Maurya, Alterations in antioxidant enzymes during aging in humans, Molecular Biotechnology 37 (2007), $58-61$.

[41] S.I. Rizvi, K.B. Pandey, R. Jha and P.K. Maurya, Ascorbate recycling by erythrocytes during aging in humans, Rejuvenation Research 12 (2009), 3-6.

42] S.I. Rizvi, M.A. Zaid, R. Anis and N. Mishra, Protective role of tea catechins against oxidation-induced damage of type 2 diabetic erythrocytes, Clinical and Experimental Pharmacology and Physiology 32 (2005), 70-75.

[43] S.I. Rizvi, R. Jha and P.K. Maurya, Erythrocyte plasma membrane redox system in human aging, Rejuvenation Research 9 (2006), 470-474.

[44] U. Cakatay, R. Kayali and H. Uzun, Relation of plasma protein oxidation parameters and paraoxonase activity in the aging population, Clinical and Experimental Medicine 8 (2008), 5157.

[45] W.J. Cai, Q.D. Cao, L. Zhu, M. Peppa, C. He and H. Vlassara, Oxidative stress-inducing carbonyl compounds from common foods: Novel mediators of cellular dysfunction, Molecular Medicine 8 (2002), 337-346.

[46] Y. Ogasawara, T. Namai, F. Yoshino, M.C. Lee and K. Ishii, Sialic acid is an essential moiety of mucin as a hydroxyl radical scavenger, FEBS Letters 581 (2007), 2473-2477.

[47] Z.Y. Wen, L.C. Song, Z.Y. Yan, Z.H. Lu, D.G. Sun, Y. Shi and S. Chien, An animal model to study erythrocytes senescence with a narrow time window of erythrocytes production: alterations in osmotic fragility and deformability of erythrocytes during their life span, Clinical Hemorheology and Microcirculation 19 (1998), 299-306. 


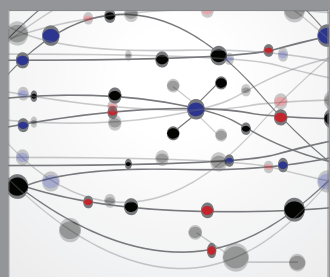

The Scientific World Journal
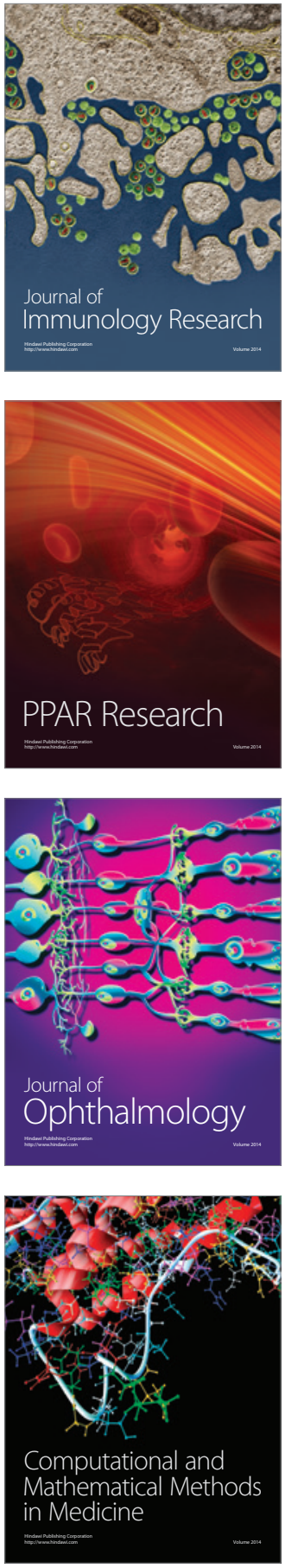

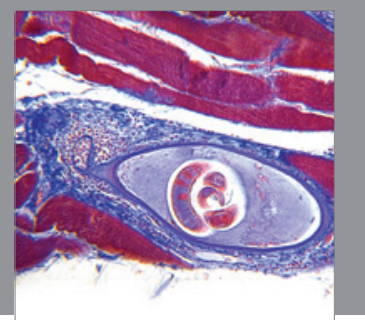

Gastroenterology

Research and Practice
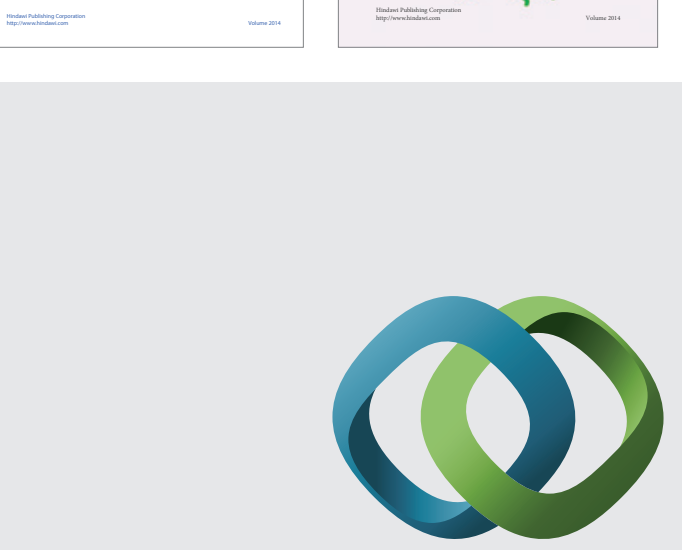

\section{Hindawi}

Submit your manuscripts at

http://www.hindawi.com
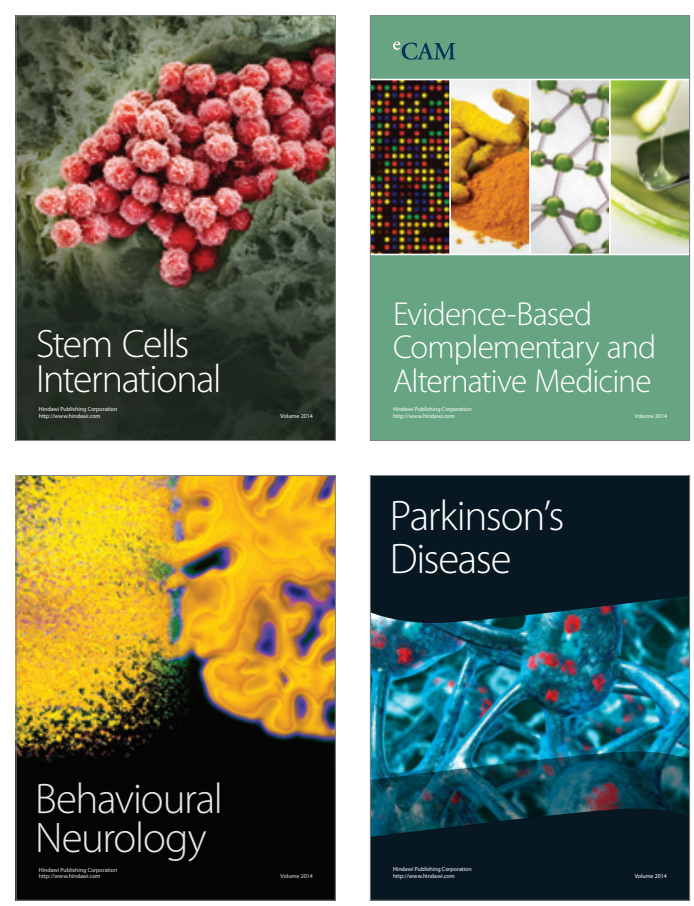

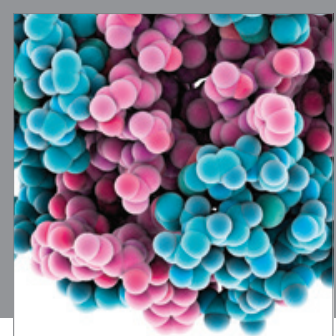

Journal of
Diabetes Research

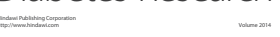

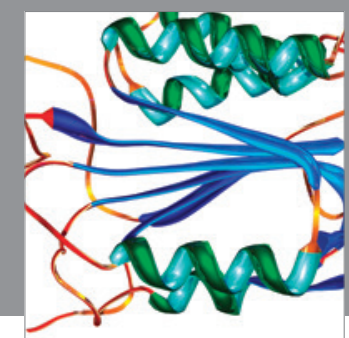

Disease Markers
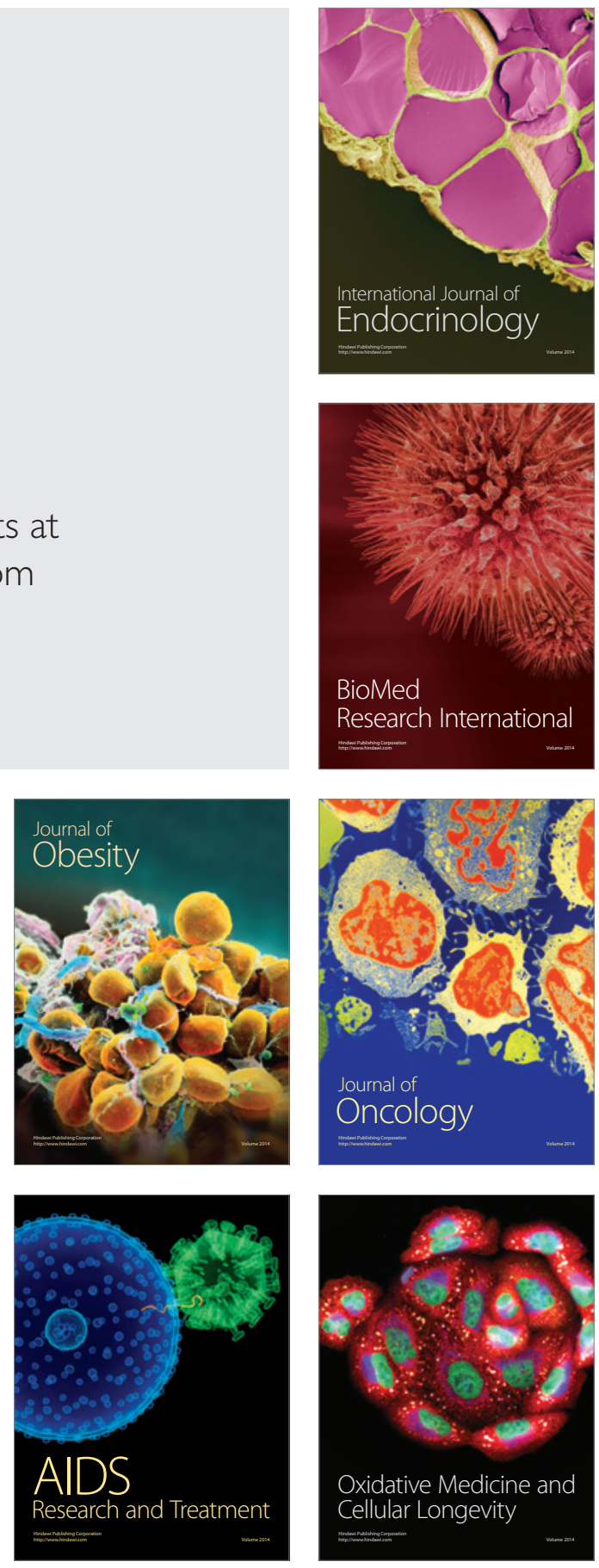